\title{
Bat-borne virus diversity, spillover and emergence
}

\author{
Michael Letko ${ }^{1,2 \bowtie}$, Stephanie N. Seifert ${ }^{1}$, Kevin J. Olival ${ }^{3}{ }^{3}$, Raina K. Plowright $\mathbb{B}^{4}$ \\ and Vincent J. Munster (10 ${ }^{1 凶}$
}

Abstract | Most viral pathogens in humans have animal origins and arose through cross-species transmission. Over the past 50 years, several viruses, including Ebola virus, Marburg virus, Nipah virus, Hendra virus, severe acute respiratory syndrome coronavirus (SARS-CoV), Middle East respiratory coronavirus (MERS-CoV) and SARS-CoV-2, have been linked back to various bat species. Despite decades of research into bats and the pathogens they carry, the fields of bat virus ecology and molecular biology are still nascent, with many questions largely unexplored, thus hindering our ability to anticipate and prepare for the next viral outbreak. In this Review, we discuss the latest advancements and understanding of bat-borne viruses, reflecting on current knowledge gaps and outlining the potential routes for future research as well as for outbreak response and prevention efforts.

'Laboratory of Virology, Division of Intramural Research, National Institute of Allergy and Infectious Diseases, Rocky Mountain Laboratories, National Institutes of Health, Hamilton, MT, USA.

${ }^{2}$ Paul G. Allen School for Global Animal Health, Washington State University, Pullman, WA, USA

${ }^{3}$ EcoHealth Alliance, New York, NY, USA.

${ }^{4}$ Department of Microbiology and Immunology, Montana State University, Bozeman, MT, USA.

凶e-mail:michael.letko@ wsu.edu; vincent.munster@ nih.gov
Bats are the second most diverse mammalian order on Earth after rodents, comprising approximately $22 \%$ of all named mammal species, and are resident on every continent except Antarctica ${ }^{1}$. Bats have been identified as natural reservoir hosts for several emerging viruses that can induce severe disease in humans, including RNA viruses such as Marburg virus, Hendra virus, Sosuga virus and Nipah virus. In addition to direct isolation of these human pathogens from bats, accumulating evidence suggests that other emerging viruses, such as Ebola viruses, severe acute respiratory syndrome coronavirus (SARS-CoV), SARS-CoV-2 and Middle East respiratory coronavirus (MERS-CoV), also originated in bats, even if other hosts, such as civets for SARS-CoV and camels for MERS-CoV, are proximate reservoirs for human infection $^{2-5}$. A growing list of emergent coronaviruses, including the Swine acute diarrhoea syndrome coronavirus, which emerged from horseshoe bats and killed $>20,000$ pigs $^{6}$, and the ongoing COVID-19 pandemic ${ }^{7}$, further underscores the ongoing threat of bat-borne viral emergence.

Bats harbour a high viral diversity relative to other mammalian orders; indeed, recent studies have suggested that viral diversity is reflective of the number of species, with Rodentia (rodents) and Chiroptera (bats) containing the most species among mammals ${ }^{8}$. This viral diversity flags bats as an important taxonomic group for global viral discovery and zoonotic disease surveillance efforts 9 . These efforts, ultimately aimed at identifying and mitigating future emergence events of bat-borne diseases, have identified thousands of novel bat-derived viral genomic sequences over the past decade. However, as most of these sequences span polymerases and not the surface proteins that often govern cellular entry, little progress has been made towards translating sequence data from novel viruses into a risk-based assessment to quantify zoonotic potential and elicit public health action. Further hampering this effort is an incomplete understanding of the animals themselves, their distributions, behaviours and interactions with the environment, and the processes that lead to contact with humans. In this Review, we discuss the current state and knowledge gaps of bat virus ecology (BOX 1) and the molecular barriers to zoonotic disease emergence; we also review advances and challenges in pandemic preparedness and provide a framework for addressing critical deficits in our understanding of bat-borne viruses.

\section{Viral diversity in bats}

Research on bat viruses dates back to the 1930s, when Joseph Pawan first identified rabies virus in bats and experimentally infected several different bat species with the virus in Trinidad ${ }^{10,11}$. The following decades saw a slow accumulation of newly discovered bat viruses ${ }^{12}$ and an exponential increase after the discovery and isolation of SARS-related coronaviruses (SARSr-CoVs) from bats in 2002 and the concomitant rise of next-generation sequencing technologies ${ }^{13,14}$. Field research on other bat-borne emerging pathogens, including Nipah virus $^{15-18}$ and Marburg virus ${ }^{19}$, in combination with the decreasing cost of next-generation sequencing technologies, spawned the current era of intensive bat viral discovery efforts. These efforts have led to the identification of whole clades of viruses and genomic sequences closely 


\section{Box 1 Bat ecology}

Our understanding of bat viral ecology has increased substantially over the past two decades, yet this knowledge remains limited to a handful of species and the extremely diverse ecology, biology and life history traits of bats pose a challenge when extrapolating data from any one species or population to bats more broadly. Beyond the within-host processes, including innate immunity and molecular interactions such as receptor compatibility, which can limit susceptibility and viral shedding, ecological factors can facilitate or inhibit virus spillover. The most revealing ecological studies thus far have used hypothesis-driven, field-sampling schemes, targeting specific reservoir hosts through time and space ${ }^{160}$. Data from these targeted, longitudinal studies, combined with mathematical modelling, have revealed that the frequency and synchronization of reproduction in a reservoir species can influence the prevalence and persistence of viruses within and between bat populations ${ }^{30,161}$. Furthermore, changes in land use, including conversion to agriculture and deforestation, can lead to nutritional stress or increased human-bat contact rates when the habitat is cleared. For example, nutritional stress is a risk factor for Hendra virus infection in flying foxes ${ }^{162}$, and Nipah virus spillover events have been linked to the consumption of contaminated date palm sap, where bats feed from the collection spigots ${ }^{163}$. Longitudinal datasets have revealed seasonal patterns in spillover of Nipah virus in Bangladesh ${ }^{164}$, of Hendra virus in Australia ${ }^{31,165,166}$, of Marburg virus in Uganda ${ }^{30}$ and of Ebola virus in central Africa ${ }^{19}$, which are related to factors such as seasonal reproductive patterns or food availability; however, the ability to predict spillover events on a finer scale remains elusive. Recent efforts to develop rigorous statistical models to prioritize surveillance for the bat species most likely to serve as reservoir hosts for key viral groups of interest ${ }^{9,167-169}$ may provide the insight necessary to maximize sampling efforts and increase the impact of field studies. Whereas environmental and host ecological factors certainly influence the risk of cross-species transmission, little is known about the fine-scale host-pathogen interactions of bats and viruses and how variation in host species and viruses at the molecular level influences broader patterns of zoonotic spillover ${ }^{34}$.

\section{Life history traits}

Traits including, but not limited

to, the timing and frequency

of reproduction, lifespan,

sex ratio and sociality.

Herd immunity

Indirect, reduced risk of infection among susceptible

individuals within a population as a result of the majority of that population having immunity through vaccination or a history of exposure or infection.

Susceptible-infectious resistant framework

A class of compartmental model used in understanding the dynamics of infectious disease in a population. related to SARS-CoV ${ }^{14}$, Swine acute diarrhoea syndrome coronavirus ${ }^{6}$, MERS- $\mathrm{CoV}^{20}$, rabies virus ${ }^{21}$, Nipah virus and Hendra virus ${ }^{22}$, new strains of influenza A virus ${ }^{23}$ and filoviruses such as Zaire Ebolavirus ${ }^{24,25}$ in various bat species. To date, thousands of new bat-associated viral species have been discovered from at least 28 diverse viral families ${ }^{26}$ (FIC. 1), the vast majority of which are likely host specific with limited zoonotic potential. For example, astroviruses are hugely diverse and can be found in $>30 \%$ of individual bats screened ${ }^{27}$, yet there are no known cases of spillover to humans of the astroviruses found in bats, although this apparent absence may also be due to the lack of active surveillance for these viruses. Other bat-associated viral families, such as coronaviruses, henipaviruses, lyssaviruses and filoviruses, are both diverse and of great public and veterinary health concern because of their rapid evolutionary rate, pathogenicity in human or other hosts and proven ability to emerge. Despite this growing field of discoveries, our knowledge of the global bat virome remains incomplete ${ }^{9,28}$. The underlying drivers of viral diversity in bat, the patterns of viral co-infection and competition dynamics, and the interactions between viruses and the bat microbiota remain largely unexplored.

New viral sequences are constantly discovered across a wide geographic range and in a growing number of bat species; however, there are few downstream studies aimed at functionally characterizing these viruses or the ecological and epidemiological factors of their hosts that may or may not facilitate zoonotic spillover. In addition, many virus discovery efforts focus on genus-level or family-level consensus PCR methods to identify close relatives of known human pathogens and sequence only a small, conserved region of the genome. Although these datasets provide valuable information for evolutionary studies and for the design of viral discovery panels for next-generation sequencing, they offer little in terms of functional characterization. Furthermore, preferentially targeting relatives of highly pathogenic viruses limits our ability to discover the next unexpected zoonotic pathogen. For example, before SARS-CoV emerged, few researchers had considered betacoronaviruses as a serious pandemic public health threat. Advances in deep sequencing make unbiased viral discovery more cost-effective and efficient for producing full-length genomes of novel viruses, thus promising a new era of bat virus genomics.

\section{Bats as unique virus hosts}

Viral persistence in bat cells and populations. Shedding of zoonotic viruses from bat populations can vary considerably in space and time ${ }^{29}$, with peaks in shedding sometimes coinciding with spillover to other species ${ }^{30,31}$. Understanding the mechanisms that drive the circulation of bat viruses in populations, including causes of peaks in shedding, is necessary to predict when and where spillover may occur. Another key requirement is understanding the biology of these infections in bats. If zoonotic infections cause a simple dynamic of acute infection followed by recovery and resistance ${ }^{32}$, as originally assumed for henipaviruses and sometimes assumed for filoviruses ${ }^{33}$, then epidemic cycles will be driven by oscillating herd immunity, connectivity, population size and other factors that drive transmission among bats ${ }^{34}$. Some observations have been inconsistent with this susceptible-infectious-resistant framework, for example, the lack of an association between high virus prevalence and large host population size $e^{35}$, whereas other observations suggest that this dynamic may be possible, for example, the short infectious periods in bats inoculated in captivity ${ }^{36}$. Other studies hint at persistent and/or recurrent infection in bats. If bats are persistently infected, immune competence may control shedding, and factors such as stress would drive shedding peaks ${ }^{34}$. For example, there is anecdotal evidence of captive bats seroconverting against Nipah virus after a period of seronegativity, suggesting that persistent infection and episodic shedding may be possible ${ }^{17,34}$. A recent observation of bats synchronously shedding multiple paramyxoviruses during a pulse of Hendra virus shedding is more easily explained by a population-level stressor affecting host immune competence; however, there could be other explanations ${ }^{37}$. Curiously, naive Rousettus aegyptiacus bats became infected with Marburg virus months after an inoculation and transmission experiment had ceased, suggesting that Marburg virus persisted within the small group of 36 experimental bats for 7 months $\mathrm{s}^{38}$. A parsimonious explanation for this is persistent infection in one or more individuals.

Within-host cycles of infection in bats have been extremely difficult to determine, and the data required to assess competing hypotheses have not yet been available. Results from inoculation experiments in bats have been difficult to interpret ${ }^{36}$, and the limited duration of almost all bat virus experiments precludes investigations 
Type I interferon

A large group of related cytokines that bind to widely expressed interferon- $a$ receptors and are responsible for regulating the immune response to infection. into viral persistence within hosts ${ }^{34}$. Ideally, genetic data from viruses infecting individually marked bats over time could be used to determine if viruses persist within individuals ${ }^{34}$, but recapturing most bats is extremely difficult, and few studies collect data longitudinally ${ }^{29}$. Recently, researchers have been able to make inferences about viral circulation in bats by fitting mathematical models of disease dynamics to longitudinal serological data. A study using such methods ${ }^{39}$ determined that persistence or reinfection of a circulating henipavirus was likely in Eidolon helvum bats. Research combining longitudinal sampling of bats with viral genomics, antibody surveys and mathematical models will be required to infer zoonotic pathogen circulation in bats ${ }^{34}$.

Intrinsic bat resistance. Bats are seemingly refractory to viral pathogenesis, and their metabolism has been at the centre of the long-standing 'flight as fever' hypothesis underlying this phenomenon ${ }^{40,41}$. Several groups have speculated that the high-energy metabolic demands of flight lead to elevated body temperatures in bats, mimicking the fever that occurs in other animals during immune activation, which may broadly impact viral pathogenesis. However, experimental studies have shown that filoviruses replicate similarly in bat cells regardless of ambient temperatures ${ }^{37,42}$. Beyond body temperature, knowledge gaps on bat reservoir species and their flight behaviour, immunity and metabolism obscure how bat metabolism relates to immunity.

Innate bat immunity. Although viruses such as Nipah virus and Marburg virus have been experimentally shown to replicate in and shed from their bat host species, a striking feature of these infections is that the bats lack overt signs of pathology $y^{36,43-45}$. The observation that bats may be refractory to, or tolerant of, viral infection was noted as early at $1936\left(\mathrm{REF}^{46}\right)$, yet the immunological mechanisms that underpin this phenotype have only begun to be elucidated in the past few years. Current data suggest that the classical pathology caused by strong activation of the immune system in response to viral infection that is seen in humans and laboratory animal models does not occur in bats ${ }^{37,47}$. The lack of pathology observed in bats is likely due to a combination of differences in viral tissue tropism and host immune responses ${ }^{48}$. Viral replication and shedding in bats in combination with an apparent lack of disease may allow for the efficient maintenance and dissemination of viruses.

Interferon-a (IFN $\alpha)$, IFN $\beta$ and IFNy pathways vary in their level of activation between bat and human cells in response to viral infection ${ }^{49-52}$. Some of these studies have shown dampened immune responses in bats, whereas others have shown heightened responses to infection. The consequences of these differences for overall pathology in bats are still to be determined. A notable finding common to all of these studies is that, regardless of the host species, all of the bat cell lines tested support filovirus infection, suggesting that the innate immune pathways assessed in these cell culture assays do not form barriers to infection.

Broader characterizations of bat innate immunity have provided some insights into the differences between bat and human immune responses. For example, Pteropus spp. bats have a substantially smaller type I interferon genomic locus than other mammals, yet they have constitutive basal expression of their IFNa genes, regardless of stimulation ${ }^{53}$. How a smaller type I interferon locus might influence viral disease is

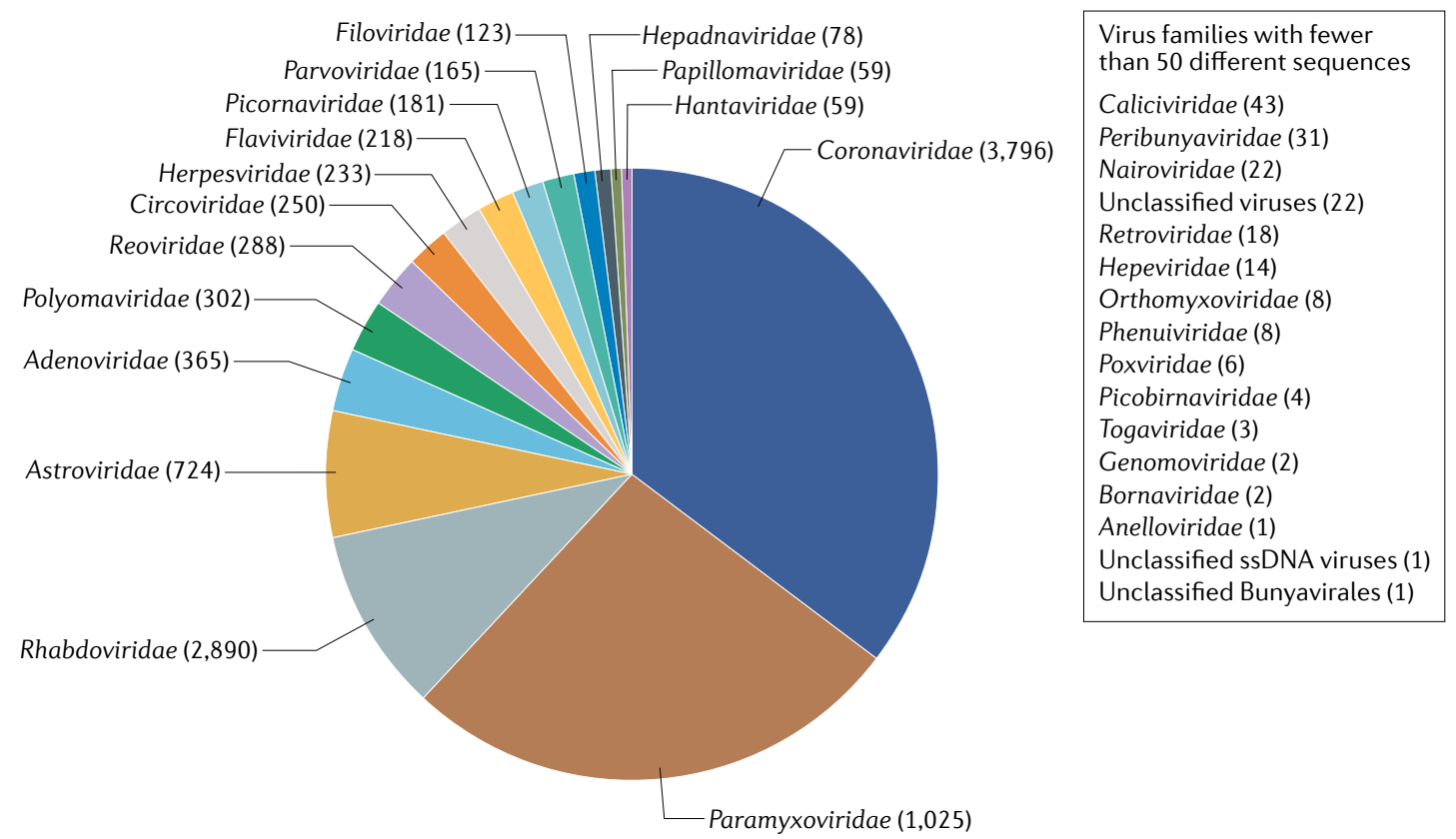

Fig. 1 | Currently described bat virus diversity. Publicly available genetic sequence data for bat-derived viruses (database of bat-associated viruses) were pooled and categorized by viral family. Of note, large parts of the bat virus diversity remain uncharacterized, and discovery efforts have prioritized virus families with known zoonotic potential such as the Coronaviridae. ssDNA, single-stranded DNA. 


\section{Box 2 | Bats as animal models}

Although a growing number of laboratories are studying infectious diseases that emerged from bats in laboratory settings, there are few examples of bat species being successfully established as animal model ${ }^{170}$. This is in part owing to the unique handling requirements for volant animals, challenges with keeping many insectivorous bats fed and healthy and difficulties in supporting complex behavioural adaptations (for example, hibernation for temperate bats). These challenges are compounded by a limited availability of breeding stock, regulations on importation of live animals and the high costs of maintaining animals in biosafety level 3 or 4 facilities, which are required for virus studies. Nevertheless, several bat species have been established as animal models, including Pteropus spp. for Nipah and Hendra virus, Eidolon helvum for African Henipaviruses, Rousettus aegyptiacus for Marburg virus, Artibeus jamaicensis for Zika virus, MERS-CoV and rabies virus and Myotis lucifugus for the fungal disease white nose syndrome ${ }^{36,43,60,120,171-174}$. These studies have led to valuable discoveries, for example, showing that $R$. aegyptiacus, from which Marburg virus was isolated, is refractory to infection with many other viruses that are associated with other bat reservoir species ${ }^{19,44,45,120,121}$, making the broad use of this bat species in disease pathology modelling questionable. Beyond the technical challenges of working with these animals, a bigger issue with bats as animal models lies in our fundamental approach to disease modelling: almost all currently established animal models in virology are centred on severe disease phenotypes and high levels of viral replication. This custom contrasts with our current understanding of bat virus biology, which assumes that bats exhibit minimal pathology and likely low levels or short temporal bursts of viral replication. Recent experimental infection studies with Tacaribe virus and Lagos bat virus in their respective natural reservoir bat species ${ }^{175,176}$ resulted in severe disease and mortality, showing that the paradigm that bats are resistant to highly pathogenic viruses should be addressed at the level of specific host-pathogen interactions rather than as a generalization for a complete animal order. Comparative studies between animal models of human disease and bat animal models are needed to understand the mechanisms responsible for the differences in disease severity of bat-borne viruses observed in natural reservoir and spillover host species.

\footnotetext{
Type III interferon

A small group of related

cytokines that bind to the interferon- $\lambda$ receptors found on epithelial cells and are responsible for regulating the immune response to infection.
}

Bat $1 \mathrm{~K}$ project

A global research initiative to sequence and annotate the genomes of all bat species, starting with more than 1,000 of the most relevant species for global health. unknown but, presumably, a smaller locus will translate into a different type of response than the one observed in other animals. Additionally, compared with Rousettus spp. bats, which have a more diverse type I interferon locus and strongly induce type I interferon in response to viral infection ${ }^{54,55}$, bats in the genus Pteropus mount a stronger type III interferon response ${ }^{56}$. However, given that there are 21 bat families, over 200 genera and 1,400 extant species of bats ${ }^{1}$, conclusions regarding general features of bat immunity and their implications for viral infection should be reserved.

Adaptive bat immunity. The adaptive humoral immune response, mediated by antibodies, is as enigmatic as the innate immune system in bats. Long-term laboratory experiments with Marburg virus challenge in the Egyptian fruit bat ${ }^{57}$ and with rabies virus challenge in the insectivorous big brown bat ${ }^{58}$ showed that, although antibodies to the respective pathogens arose, they rapidly waned below detectable levels after $\sim 3$ and 5 months, respectively. Although bats had low levels of antibodies to Marburg virus 22 months after the initial challenge, re-challenge resulted in dramatically reduced dissemination and viral spread ${ }^{57}$. Interestingly, $R$. aegyptiacus challenged with Ebola virus, Marburg virus or Sosuga virus generated detectable antibody responses post challenge, but none of the antisera could neutralize the live virus, suggesting that antibody-dependent cell-mediated responses are crucial in the viral clearance of these viruses $^{59}$. By contrast, bats generate virus-neutralizing antibodies to Nipah virus ${ }^{60}$ and rabies virus ${ }^{61}$ upon experimental challenge; however, live virus may be concurrently detected in saliva or urine, suggesting incomplete virus clearance. Curiously, Sosuga virus and Nipah virus are both paramyxoviruses and yet elicit different types of antibody responses - the mechanisms and underlying reasons are unclear. Recent studies support the notion that the immune response in bats is adapted for pathogen tolerance and regulated to mitigate immunopathology, potentially promoting incomplete viral clearance and asymptomatic infection ${ }^{55,62}$. Undoubtedly, the complex evolutionary history between bats and their viruses plays a role in how and when the bat immune system is stimulated in response to viral infection; thus, broad conclusions from single pathogen and single host studies should remain tempered.

Challenges of studying viral infections in bats. A major problem facing all studies of bat-derived virus cell biology is the lack of available reagents and animal models (BOX 2), compounded by the enormous taxonomic diversity of these animals. Most mammalian cell lines currently available are not derived from bats and do not support replication of the majority of viruses being discovered. For example, the isolation of bat-derived coronaviruses has been challenging owing to a limited ability to infect the standard, typically primate-derived, cell lines used in most laboratories ${ }^{14,63,64}$. Even cell lines derived from the same bat species that the viruses were originally sequenced from may fail to support replication owing to loss of expression of the host receptor ${ }^{65}$. Thus, there is an urgent need for more cell culture reagents that can better facilitate virus isolation, either new cell lines capable of supporting replication of bat viruses or genetically modified versions of existing cells, such as Vero cells, to increase their susceptibility to viral infection with bat viruses. Ideally, these new cell lines should be derived from a wide range of species and tissues. In addition, organoid systems incorporating multiple cell types within a 3D architecture to reproduce tissue-specific functional properties could potentially facilitate the translation from in vitro single-cell type studies to organ-specific host-pathogen interaction studies. Lastly, live animal models will also be crucial for understanding the implications of molecular findings in bat cells for the course of infection in the natural host.

Whereas large-scale CRISPR-Cas9-mediated knockout and activation screens have been valuable for identifying human proteins involved in viral infection, they have yet to be applied to other host species, including bats. Given that an annotated transcriptome is now available for the R. aegyptiacus bat ${ }^{66}$, similar screens could be performed in Rousettus cells to elucidate the factors involved in filovirus infection, for example. The ambitious Bat $1 \mathrm{~K}$ project has begun the process of generating genome sequence data for all extant bat species ${ }^{67}$, and the results of this effort will undoubtedly be an invaluable resource in elucidating specific bat-pathogen interactions.

Many of the earliest studies of bat immunity focused on the humoral adaptive immune response in bats, yet we still lack an understanding of the regulation of B cell proliferation, affinity maturation and the mechanisms 
Monophyletic

Multiple organisms all derived from a single common ancestor. underlying the transitory nature of bat antibody responses. Countless studies have conducted serological surveillance for antibodies to specific viruses in wild bats; however, there is relatively little experimental data with bats as a model system, particularly spanning longer periods of time. The information available from field-collected samples is limited by the unknown history of sampled bats with regard to their reproductive status, age, sex, nutritional status and infection status, with any number of pathogens potentially influencing the immune response.

\section{Viral spillover from bats}

The spillover of bat-associated viruses requires a combination of factors, including ecological opportunity for contact, virus-host molecular and cellular compatibility, and a permissive or circumvented immune response, as described in detail herein. Yet, despite the various potential barriers and the fact that many spillover events may go undetected by surveillance systems, there is a growing list of recent bat-borne zoonotic spillover events. This list includes examples of direct bat-to-human spillover, which are supported by both epidemiological evidence and molecular detection of monophyletic viruses between bat and human populations; the examples include near annual outbreaks of Nipah virus in Bangladesh since 2001 (REF. ${ }^{68}$ ), several Marburg virus outbreaks across Africa $^{2}$ and outbreaks of rabies virus and other novel Lyssaviruses globally. Other examples of indirect bat-to-human spillover that involve intermediate hosts are also supported by epidemiological and molecular

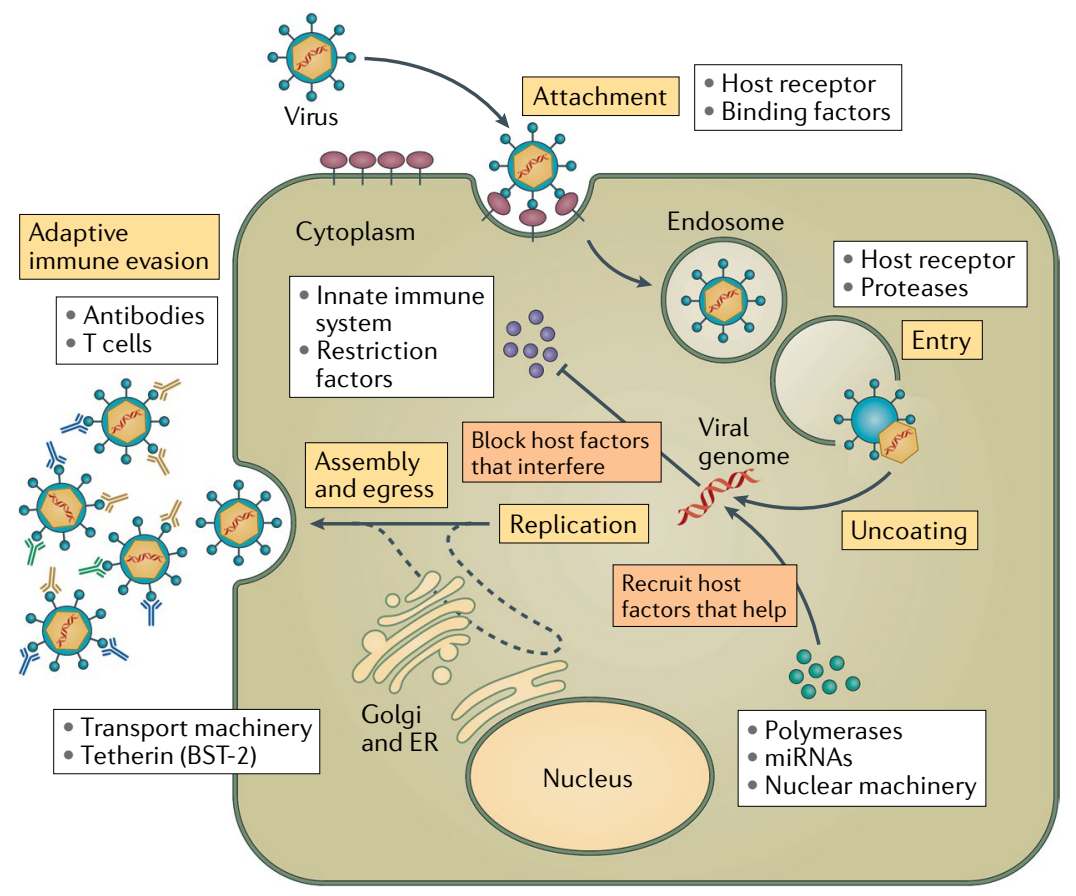

Fig. 2 | Overview of molecular host species barriers. Viruses rely on numerous interactions with the host cell machinery in order to replicate and transmit. Virus-host interactions occur at every stage of the viral life cycle, including attachment, uncoating, genomic replication and protein expression, viral assembly and egress. While many factors involved are still unknown, some well-described examples are indicated. ER, endoplasmic reticulum; miRNA, microRNA. evidence, including Hendra virus in 1994 via horses ${ }^{69}$ and Nipah virus in Malaysia in 1997 and 1998 via pigs $^{70}$. The SARS-CoV 2002-2003 outbreak in southern China and the 2019 SARS-CoV-2 emergence in central China were retrospectively connected to bat populations via molecular evidence and appeared to involve intermediate hosts. Several viruses closely related to SARS-CoV were found in bats, and actual SARS-CoV was directly isolated from animals in open-air markets ${ }^{14,63,71}$. Analogously, several bat viruses have now been identified to have a high similarity to SARS-CoV-2 (REF. $\left.{ }^{72}\right)$. In several other cases, bat-to-human spillover was assessed retrospectively via serological human cohort studies; for example, Henipavirus spillover among bat hunters in Cameroon ${ }^{73}$, bat-borne reovirus (Melaka virus and Pulau virus) exposure in people living in close proximity to bat roosts on Tioman Island, Malaysia ${ }^{74}$, as well as in a random sample screened in Singapore ${ }^{75}$, Filovirus exposure of bat hunters in India ${ }^{76}$ and ongoing human exposure to SARSr-CoVs in rural communities in China occurring after the 2003 SARS outbreak ${ }^{77}$.

\section{Molecular biology of transmission}

All viruses, regardless of classification or origins, must be able to subvert and overcome various molecular factors within their hosts in order to replicate and spill over into new species. Every stage of the viral life cycle relies on numerous protein interactions with the host cell, including viral binding and entry, recruitment of host factors essential for viral replication, suppression of antiviral host factors, assembly and egress from the cell, and evasion of the host immune system ${ }^{78}$ (FIG. 2). Viral-host protein interfaces have been shown, through functional and structural studies, to be remarkably specific and involve multiple points of $\operatorname{contact}^{79}$. Even single amino acid variations can impact or abrogate a viral-host protein interaction between different species and form a molecular block, or species barrier, to viral replication ${ }^{80-85}$. The complexity of viral-host protein interactions is compounded by how many interactions occur during any given infection. Recent proteomics studies have identified at least 194 protein interactions between Ebola virus and human host cells ${ }^{86}, 198$ virus-host protein interactions for Zika virus ${ }^{87}, 101$ for Nipah virus ${ }^{88}$ and over 300 for influenza A virus ${ }^{89}$. Given that even small perturbations in these complex networks of virus-host interactions can make the difference between a dead-end infection or viral emergence in a new host species, it is likely that the majority of bat-borne viruses fail to infect novel species as a result of within-host barriers ${ }^{90,91}$.

Cell entry of zoonotic viruses. One of the first major virus-host protein interactions that occurs during the course of infection is at the level of viral cell entry, when the virus interacts with the host receptor to facilitate the release of viral components into the cytoplasm. Depending on the virus, this process can involve one or more viral proteins, one or more host components and encompass several steps occurring at the cell surface or at an internalized membrane.

It is not surprising that many bat-borne zoonotic viruses have evolved to use highly conserved host 
molecules for cell entry that have little genetic variation between different species. Henipaviruses bind to the ephrin family of signalling proteins ${ }^{92-94}$, Filoviruses bind to the cholesterol transporter Niemann-pick C1 (NPC1) $)^{95,96}$ and Betacoronaviruses have been shown to bind different common cell-surface proteases, including angiotensin-converting enzyme 2 (ACE2) in the case of SARS-CoV and SARS-CoV-2 (REF. ${ }^{97}$ ) and dipeptidyl peptidase IV (DPP4) in the case of MERS-CoV ${ }^{98}$. Indeed, these receptors are nearly identical, at least in the regions that interact with the virus, between various bat species, intermediate host species, such as camels and palm civets, and humans ${ }^{84,85,99}$.

Restriction of cell entry is not easily overcome by viruses. For example, wild-type mice are completely resistant to infection with MERS-CoV because of differences in murine DPP4 glycosylation from human DPP4 (REF. $\left.{ }^{100}\right)$. Despite great effort from the animal disease-modelling community, to date, there is no MERS-CoV isolate that can use wild-type murine DPP4, likely because too many viral adaptations are necessary. However, partial blocks to cell entry are more easily overcome. Recently, it has been shown that MERS-CoV can rapidly acquire single-point mutations to increase its compatibility with DPP4 from different bat species ${ }^{85}$. Some MERS-related CoVs discovered in bats, which appear nearly identical to MERS-CoV over most of the genome, have mutations at key binding sites across the receptor-binding spike glycoprotein and are thus unable to bind to human DPP4 (REF. ${ }^{64}$ ). Similar types of viral adaptation have been observed for other zoonotic viruses such as SARS-CoV ${ }^{101}$, parvoviruses ${ }^{102}$ and avian influenza $A$ virus ${ }^{103}$. Taken together, the ability to use conserved host receptors and readily adapt to receptor variation between species are two hallmarks of viruses that have spilled over into the human population.

The genetic diversity of many RNA viruses can be attributed to high mutation rates, short generation times and the strong selective pressure of the host environment (during natural infections or after vaccinations); however, positive-stranded RNA viruses, including SARS-CoV and MERS-CoV, have relatively low mutation rates associated with $3^{\prime}-5^{\prime}$ exoribonuclease proofreading activity ${ }^{104-108}$. The rapid evolution of coronaviruses to the host environment is largely driven by the high rates of genetic recombination, which facilitate the acquisition of multiple mutations in a single event. Inheriting multiple genetic changes at once can have dramatic effects on viral replication and subsequent adaption to new host environments. Recent phylogenetic analyses have revealed that the variation in MERS-CoV circulating in camel populations is largely driven by recombination ${ }^{109-112}$. Although SARSr-CoVs have been identified and isolated from bats, no single bat isolate perfectly matches the human strains. For example, some SARSr-CoVs can use the human receptor but vary drastically from SARS-CoV in the $3^{\prime}$ end of their genome, whereas other SARSr-CoVs are nearly identical to SARS-CoV in this region but fail to interact with the human receptor ${ }^{113}$. In further support of these findings, the entire SARS-CoV genome has now been sequenced across multiple separate but related viruses circulating in bats, strongly suggesting that the human virus is a recombinant form of these ancestral variants ${ }^{114}$. Outside coronaviruses, recombination in the rabies virus glycoprotein was shown to facilitate cross-species transmission from bats to skunks and raccoons ${ }^{115}$. Thus, in addition to the rapid mutation rate characteristic of many RNA viruses, recombination provides an additional mechanism to rapidly overcome barriers in novel host species.

Post-entry virus-host interactions of zoonotic viruses. Whereas our understanding of viral entry as a species barrier is becoming clearer for many emerging zoonotic viruses, cellular blocks beyond entry are more elusive and remain largely unknown. However, research over the past 20 years with well-studied zoonotic pathogens, such as lentiviruses, including HIV and its evolutionary predecessor simian immunodeficiency virus, and influenza A virus, which includes avian influenza $A$ virus, has led to the identification of numerous human and primate intracellular species barriers in the form of dependency factors, which these viruses rely on to replicate, and restriction factors, which are antiviral proteins that specifically interfere with viral replication ${ }^{116}$. For example, the ability of the simian immunodeficiency virus accessory protein Vif to antagonize the host restriction factor APOBEC3 is vital in determining the potential host breadth in non-human primates ${ }^{117,118}$. The avian influenza A virus accessory protein PB1-F2 disrupts mitochondrial antiviral signalling more efficiently in the avian host than the truncated PB1-F2 common in mammalian influenza A viruses ${ }^{119}$. Although the post-entry species barriers that limit host breadth for lentiviruses and influenza viruses are likely different from those that limit host breadth for the emerging bat-borne infectious diseases, the research framework for these well-studied host-pathogen systems can serve as a roadmap for research into bat-borne host-pathogen systems.

Recent experimental infection studies in bats provided evidence of species-level post-entry barriers, suggesting that some bat-borne viruses are likely host specific and may have a limited ability to transmit between certain bat species. For example, the Rhinolophus spp. bat coronavirus WIV1-CoV and Ebola virus can use the cellular receptors from $R$. aegyptiacus bats to enter cell lines, but these bats fail to support any WIV1-CoV infection and only poorly support Ebola virus replication ${ }^{84,120,121}$. Transcriptomics studies have identified several immune signalling pathways that are activated differently in human versus Rousettus spp. cells $s^{51,52}$. Other studies have taken more direct approaches to identify post-entry barriers to replication. Mass spectrometry has pinpointed the host E3-ubiquitin ligase, RBBBP6, as a negative regulator of Ebola virus transcription, which functions by binding VP30, a viral protein that is key in replication ${ }^{86}$. A similar study found that the key Ebola virus protein involved in antagonizing the host interferon pathway, VP35, forms an essential interaction with host TRIM6 protein and that the disruption of this interface reduces viral replication ${ }^{122}$. Additionally, tetherin ${ }^{123-125}$ and IFITM host proteins ${ }^{126}$, 

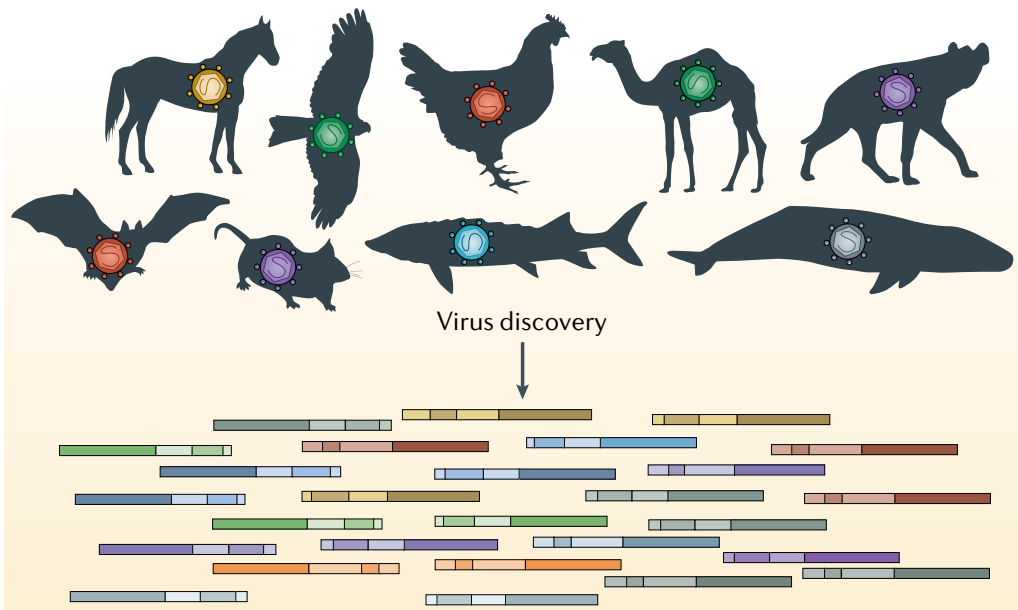

Current viromics research

Future viromics research

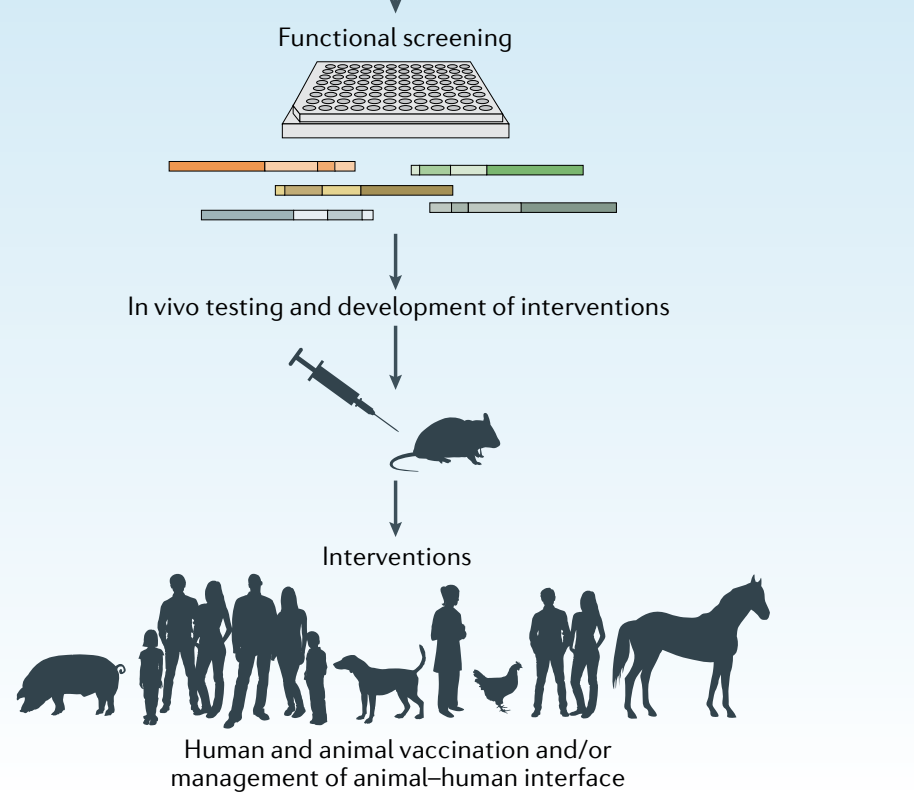

Fig. 3 | Using functional viromics to move beyond zoonotic virus discovery. Current viromics research, mostly based on the targeted amplification of viral sequences or sometimes metagenomics, stops after the identification of novel viral sequences in animals. Large-scale functional screens of viruses in vitro will facilitate transmission and pathogenesis studies in vivo and ultimately lead to the development of 'One Health' intervention strategies, such as vaccination of humans or reservoirs or intermediate animal hosts, as well as other measures to reduce the risk of contact and viral transmission at the animal-human interface.

\section{Viral pseudotype}

A genetically modified virus

that has incorporated the glycoprotein of another virus

and can produce a measurable reporter, such as GFP or

luciferase, upon infection of a cell. which have been shown to inhibit lentiviruses and influenza A virus, respectively, have broad antiviral effects to Ebola virus and SARS-CoV. Tetherin from fruit bats inhibits Nipah virus but not Ebola virus ${ }^{127}$. Compared to more well-studied viruses with a zoonotic origin, such as influenza A virus and HIV, there is still much to uncover for post-entry species barriers of emergent, bat-derived viral pathogens. Large-scale CRISPR-Cas9-mediated knockout and activation screens in human cells have recently identified specific host factors that are essential to flaviviruses ${ }^{128-130}, \mathrm{HIV}^{131}$, Epstein-Barr virus ${ }^{132}$ and influenza A virus ${ }^{133,134}$ but such data have not yet been generated for bat-derived viruses.

\section{Moving beyond virus discovery}

Worldwide consortiums such as the USAID PREDICT programme as well as many independent academic laboratories around the world have used a combination of consensus PCR screening and deep sequencing to characterize thousands of novel viral sequences in samples taken from healthy bats ${ }^{135}$. Many of these novel viruses, or viral fragments, are phylogenetically related to pathogens of interest to public health; however, the capacity for these novel viruses to cause future outbreaks typically remains unresolved. With viruses isolated or sequenced directly from bat samples and molecular approaches, including viral pseudotype studies and reverse genetics, researchers have been able to demonstrate the potential of novel viruses to replicate in human cells or use human receptors for entry - the first step to incorporate virus discovery into a more comprehensive risk-reduction framework $^{14,63,136}$ (FIG. 3). Characterizing the breadth of naturally occurring bat-borne viruses, including close relatives to human viruses, is critical and leads to important insights. For example, the discovery and subsequent investigations of a novel non-pathogenic henipavirus ${ }^{137}$, Cedar virus, related to Nipah virus and Hendra virus, have proved invaluable in revealing the genetic determinants of pathogenicity in henipaviruses. Unlike Nipah virus, Cedar virus does not produce $\mathrm{V}$ or $\mathrm{W}$ proteins, which are responsible for antagonizing the host interferon pathway, and it also relies on different host cell receptors ${ }^{138}$. The identification of viruses related to Ebola virus in various bat species, including the novel Bombali virus, has provided additional support for bats as reservoirs for Ebola viruses and, even though there are no reports of filovirus haemorrhagic fever in China, filoviruses have also been identified in Rousettus spp. bats in China ${ }^{139}$. Thus, further research is needed to confirm host species range and the potential for human infection ${ }^{25}$. Ancestral variants of zoonotic coronaviruses similar to SARS-CoV, MERS-CoV and SARS-CoV-2 have been identified from bats, with some viruses related to SARS-CoV even being capable of using the human receptor ACE2 (REFS ${ }^{14,63,72,114,140}$ ). Although both SARS-CoV and MERS-CoV have also been isolated from intermediate hosts (palm civets ${ }^{141}$ and camels ${ }^{142}$, respectively), it is possible that these two viruses can be directly transmitted to humans from bats.

\section{Linking surveillance and control}

Although whole genome data are needed for downstream and comparative studies, even knowing the taxonomic family of a novel pathogen causing an outbreak can help narrow down control and treatment strategies. Novel diagnostic platforms such as the GeneXpert ${ }^{\mathrm{TM}}$ (Cepheid Inc.), which is a semi-automated PCR-based test, enable rapid, multiplexed detection of a wide panel of human pathogens and are constantly being improved to increase sensitivity and pathogen coverage. Virus discovery efforts are producing a wealth of genome sequencing data that are then made publicly available through online data repositories. Pan-serological assays are also facilitating virus discovery and providing insights into antibody cross-reactivity ${ }^{77}$. The resulting datasets provide insight into the existing variation in 
Reverse genetics

Experimentally introducing mutations into the genetic sequence of proteins or whole virus and subsequently testing for the effect on various phenotypes either in cell culture or in animals. viral families, allowing for the development of diagnostic and surveillance assays broadly targeting virus clades. For example, Bombali ebolavirus was initially discovered in bat samples using a consensus PCR assay ${ }^{25}$ developed to target a region of the viral genome for which the nucleic acid sequence is conserved between related viruses. The identification of key reservoir species and the development of better models to predict virus spillover events could enable targeted prophylactic vaccination campaigns of humans and potential reservoir hosts or intervention strategies to minimize contact between bats and humans. For example, high-risk populations that geographically overlap and have high levels of contact with bats and other wildlife could be vaccinated against Ebola virus to prevent outbreaks rather than responding to them after spillover, and local communities could benefit from education campaigns on how to live safely around bats and reduce direct contact ${ }^{143}$. Alongside these measures, efforts should be taken to reduce bat habitat destruction, which results in increased contact between bats and humans and is considered a cause of viral spillover ${ }^{144}$.

Next-generation vaccine technologies are platforms that are broadly and rapidly adaptable for different types of viral pathogens. Importantly, several of these platforms use genetically modified viruses, such as the vesicular stomatitis virus (VSV) and ChadOxl platforms, which can induce protective immunity in humans, mice, guinea pigs, non-human primates and livestock ${ }^{145-147}$ to a number of pathogens, including bat-borne Ebola virus and Nipah virus. Vaccine efficacy in animals, for example, horse vaccination for Hendra virus, including livestock and other peri-domestic animals, may even enable proactive measures to reduce cross-species transmission of bat viruses to humans ${ }^{148}$. Although still nascent and only in early clinical trials, novel platforms such as

\section{Box $3 \mid$ The future of bat virus research}

There is a growing anxiety in the field that merely identifying all the novel animal-derived viruses will do little to prevent the next outbreak. This is in part due to the near total lack of downstream assays to functionally characterize these viruses at the scale at which they are discovered. Thus, most studies have focused on animal viruses that already bear close resemblance to known human pathogens. One avenue for future research efforts should focus on the development of scalable tools that can functionally assess important questions related to viral zoonosis such as whether or not novel bat viruses can infect human cells or use known human receptors. With the cost of gene synthesis decreasing as the technology advances, novel viral glycoprotein sequences could be synthesized in bulk and tested in vitro, for example. Recently, we described a platform to test coronaviruses in bulk scale for their receptor usage ${ }^{140}$. Beyond functional studies, disease ecology and modelling are essential to determine the true risk of cross-species transmission, as are better collaborations between bat biologists and disease experts ${ }^{177}$. Recent advances in the miniaturization of device technology have produced smaller GPS trackers and more efficient, higher resolution camera traps that will certainly help improve our understanding of host species distribution and key interactions at the bat-human-environment interface. These devices are slowly being deployed in the field to increase our understanding of bat migratory patterns and bat-environment interactions. New weather and environmental satellites are providing a finer resolution of global climate trends, urbanization and development. In addition, open-access datasets of host-virus associations combined with new analytical approaches, for example, machine learning, are expanding our understanding of virus host ranges beyond the limits of current surveillance data ${ }^{9,168}$. Collectively, such information is advancing our ability to pinpoint potential hotspots of zoonotic spillover and identify new host reservoirs.
DNA-based and mRNA vaccines offer the potential for an incredibly rapid response time from pathogen discovery to therapeutic intervention. Using these technologies, researchers were able to test the first Zika vaccine in mice and non-human primates within 3.5 months of the initial outbreak in 2015 and, more recently, a similar RNA-based vaccine was designed for SARS-CoV-2 and entered human clinical trials only 2 months after the virus sequence was published ${ }^{149}$. Other platforms based on VSV or adenovirus are already FDA approved and have been shown to be effective in several species and for most of the major emerging viruses identified to date.

Depending on the route of transmission, pre-emptive control strategies may also include low-cost, lowtechnology (that is, ecological) countermeasures. For example, Nipah virus is believed to be transmitted to humans through date palm sap collection containers that have been contaminated with virus-containing urine from visiting fruit bats ${ }^{150}$. One proposed intervention is covering the containers to prevent bat feeding and contamination with bat urine ${ }^{151}$. Proactive wildlife and livestock mortality surveillance, such as great ape Ebola virus carcass surveillance in the Republic of the Congo, could function as an early warning system preceding spillover to the human population ${ }^{152}$.

Challenges to outbreak control. 'One Health' approaches involve addressing zoonosis at the human, animal and environmental levels. One of the biggest hurdles to preventing zoonosis at the animal level is the limited feasibility of wildlife vaccination. Given that filoviruses and coronaviruses are likely hosted in a variety of different animal populations, including multiple bat species and other mammals, covering large geographic regions, current vaccination delivery methods are impracticable and likely insufficient to induce effective herd immunity. Although effective vaccines are now in development for Ebola virus, Hendra virus and rabies virus, there are no effective vaccines currently available for both human and animal use. Some progress has been made on this front, for example, in the form of oral vaccines against rabies in $\operatorname{dog} \mathrm{s}^{153}$ and bats ${ }^{154}$ as well as against plague in black-tailed prairie dogs ${ }^{155}$. Applying similar efforts to other viruses in lesser-studied and more remote bat populations and other mammals will require a greater understanding of host ecology and behaviour.

After it was discovered that horses are susceptible to Hendra virus and can amplify the virus and infect humans, the Australian government invested in developing a highly effective vaccine that could be given to horses ${ }^{156}$. It was hoped that reducing transmission of the virus to horses would reduce transmission to humans. However, vaccination efforts in Australia have been hindered by antivaccination sentiment, the public perception of the vaccine being too costly for such a rare pathogen and by anecdotal evidence of unwanted side effects. This lack of adoption of Hendra virus vaccination has allowed for sporadic Hendra virus outbreaks in horses to continue ${ }^{157}$, threatening human and animal health.

The geopolitical climate represents an even bigger challenge to outbreak prevention. Despite the existence of multiple, experimental therapeutic options, the latest 
Ebola virus outbreak in the Democratic Republic of Congo (ongoing since 2018) has been stymied by civil war breaking down the health-care system and militant groups targeting health-care workers and outbreak response teams ${ }^{158}$. The recombinant VSV Ebola virus vaccine has been successfully used in response to Ebola virus outbreaks and is now FDA approved. The full licensure of the VSV vaccine will allow for a broader pre-emptive rather than reactive vaccination approach and marks the first licensure of a human vaccine for a bat-borne infectious disease since rabies. Another example of geopolitical disruption was seen during the emergence of SARS-CoV in China in 2002, when the Chinese government delayed reporting the health crisis to the international community well after the outbreak had begun to spread $^{159}$. The timely release of public health data remains a critical issue during the current COVID-19 pandemic and appears to have improved with the release of case data and full genome viral sequences as the outbreak develops (novel 2019 coronavirus on virological.org).

\section{Conclusions}

Disease $\mathrm{X}$, or the as yet unknown pathogen poised to cause the next pandemic, poses a grand challenge in outbreak prevention and response. Bats represent an important but largely uncharacterized source of known human pathogens. Despite a limited understanding of bats, the viruses they carry, and the molecular and ecological forces driving viral spillover, the tools to develop next-generation vaccines and antiviral technologies are maturing such that researchers will be able to respond to the next outbreak with unprecedented speed. In order to transition bat virus research from reactive to predictive with the ability to determine which pathogens represent the greatest threat to global health, vast advancements are needed across a multitude of disciplines (BOX 3). Here, we have identified the important progress made in the past decade and the gaps remaining in our understanding of bat virus ecology, genetic diversity and the molecular mechanisms underlying zoonotic infection and immunity. The emergence and re-emergence of zoonotic bat pathogens demonstrates the inextricable link between the health of humans, animals and the environment. Therefore, efforts to mitigate the public health impacts of bat-borne viruses must integrate research across these disciplines, applying a 'One Health' approach, from field to lab, to address the problem. The future of bat virus research lies in a combined and concerted effort to evaluate the molecular and macro-ecological risk factors of transmission, shine light on which viruses carry the potential to spill over and conduct large-scale, longitudinal surveillance studies that will support the deployment and evaluation of next-generation interventions. Nevertheless, the emergence of SARS-CoV-2 continues to present new and sobering challenges. The ongoing COVID-19 pandemic has clearly demonstrated that a dramatic increase in knowledge on pathogen emergence combined with a rapid all-out aggressive response aimed at tracing zoonotic spillover events is needed to prevent the repetition of current events.

Published online 11 June 2020
1. Burgin, C. J., Colella, J. P., Kahn, P. L. \& Upham, N. S. How many species of mammals are there? J. Mammalogy 99, 1-14 (2018).

2. Olival, K. J. \& Hayman, D. T. Filoviruses in bats: current knowledge and future directions. Viruses 6 , 1759-1788 (2014)

3. Memish, Z. A. et al. Middle East respiratory syndrome coronavirus in bats, Saudi Arabia. Emerg. Infect. Dis. 19, 1819-1823 (2013)

4. Leroy, E. M. et al. Fruit bats as reservoirs of Ebola virus. Nature 438, 575-576 (2005). The first study to report a partial Ebola virus genomic sequence from a survey of African fruit bats.

5. Amman, B. R. et al. A Recently discovered pathogenic paramyxovirus, Sosuga virus, is present in Rousettus aegyptiacus fruit bats at multiple locations in Uganda. J. Wildl. Dis. 51, 774-779 (2015).

6. Zhou, P. et al. Fatal swine acute diarrhoea syndrome caused by an HKU2-related coronavirus of bat origin. Nature 556, 255-258 (2018).

Demonstrates how a coronavirus transmitted from bats to pigs, leading to a severe outbreak that impacted the global pig industry.

7. Jiang, S., Xia, S., Ying, T. \& Lu, L. A novel coronavirus (2019-nCoV) causing pneumonia-associated respiratory syndrome. Cell. Mol. Immunol. 17, 554 (2020).

8. Mollentze, N. \& Streicker, D. G. Viral zoonotic risk is homogenous among taxonomic orders of mammalian and avian reservoir hosts. Proc. Natl Acad. Sci. USA 117, 9423-9430 (2020).

9. Olival, K. J. et al. Host and viral traits predict zoonotic spillover from mammals. Nature 546, 646-650 (2017).

10. Pawan, M. B. The transmission of paralytic rabies in trinidad by the vampire bat (Desmodus Rotundus Murinus Wagner, 1840). Ann. Trop. Med. Parasitol. 30, 101-103 (1936)

11. Hurst, E. W. \& Pawan, M. B. An outbreak of rabies in Trinidad without history of bites, and with the symptoms of acute ascending myelitis. Lancet $\mathbf{2 1 8}$, 622-628 (1931)
12. Calisher, C. H., Childs, J. E., Field, H. E., Holmes, K. V. $\&$ Schountz, T. Bats: important reservoir hosts of emerging viruses. Clin. Microbiol. Rev. 19, 531-545 (2006).

13. Li, W. et al. Bats are natural reservoirs of SARS-like coronaviruses. Science 310, 676-679 (2005). First evidence of lineage $B$ betacoronaviruses circulating in bats.

14. Ge, X. Y. et al. Isolation and characterization of a bat SARS-like coronavirus that uses the ACE2 receptor. Nature 503, 535-538 (2013).

Shows that a lineage B betacoronavirus from bats can directly use the human receptor, further providing evidence that an intermediate host may not be necessary for coronavirus spillover into humans

15. Anthony, S. J. et al. A strategy to estimate unknown viral diversity in mammals. mBio 4, e00598-13 (2013).

16. Chua, K. B. et al. Isolation of Nipah virus from Malaysian Island flying-foxes. Microbes Infect. 4, 145-151 (2002)

17. Rahman, S. A. et al. Characterization of Nipah virus from naturally infected Pteropus vampyrus bats, Malaysia. Emerg. Infect. Dis. 16, 1990-1993 (2010).

18. Reynes, J. M. et al. Nipah virus in Lyle's flying foxes, Cambodia. Emerg. Infect. Dis. 11, 1042-1047 (2005).

19. Towner, J. S. et al. Isolation of genetically diverse Marburg viruses from Egyptian fruit bats. PLoS Pathog 5, e1000536 (2009). First isolation of a pathogenic human filovirus from wild bats.

20. Wang, Q. et al. Bat origins of MERS-CoV supported by bat coronavirus HKU4 usage of human receptor CD26. Cell Host Microbe 16, 328-337 (2014).

21. Marston, D. A. et al. Complete genome sequence of Lleida Bat Lyssavirus. Genome Announc. https://doi.org 10.1128/genomeA.01427-16 (2017).

22. Drexler, J. F. et al. Henipavirus RNA in African bats PLoS One 4, e6367 (2009).

23. Tong, S. et al. New world bats harbor diverse influenza A viruses. PLoS Pathog. 9, e 1003657 (2013).
24. Yang, X. L. et al. Characterization of a filovirus (Mengla virus) from Rousettus bats in China. Nat. Microbiol. 4, 390-395 (2019).

25. Goldstein, T. et al. The discovery of Bombali virus adds further support for bats as hosts of ebolaviruses. Nat. Microbiol. 3, 1084-1089 (2018).

26. Chen, L., Liu, B., Yang, J. \& Jin, Q. DBatVir: the database of bat-associated viruses. Database 2014 , bau021 (2014).

27. Young, C. C. \& Olival, K. J. Optimizing viral discovery in bats. PLoS One 11, e0149237 (2016).

28. Carroll, D. et al. The global virome project. Science 359, 872-874 (2018)

29. Becker, D. J., Crowley, D. E., Washburne, A. D. \& Plowright, R. K. Temporal and spatial limitations in global surveillance for bat filoviruses and henipaviruses. Biol. Lett. 15, 20190423 (2019).

30. Amman, B. R. et al. Seasonal pulses of Marburg virus circulation in juvenile Rousettus aegyptiacus bats coincide with periods of increased risk of human infection. PLoS Pathog. 8, e1002877 (2012).

31. Plowright, R. K. et al. Ecological dynamics of emerging bat virus spillover. Proc. Biol. Sci. 282, 20142124 (2015).

32. Plowright, R. K. et al. Urban habituation, ecological connectivity and epidemic dampening: the emergence of Hendra virus from flying foxes (Pteropus spp.). Proc. Biol. Sci. 278, 3703-3712 (2011). This study synthesizes across disciplines and reveals the complex factors leading to Hendra virus spillover from flying foxes in Australia.

33. Hayman, D. T. Biannual birth pulses allow filoviruses to persist in bat populations. Proc. Biol. Sci. 282, 20142591 (2015).

34. Plowright, R. K. et al. Transmission or within-host dynamics driving pulses of zoonotic viruses in reservoir-host populations. PLoS Negl. Trop. Dis. 10, e0004796 (2016)

35. Giles, J. R. et al. Environmental drivers of spatiotemporal foraging intensity in fruit bats and implications for Hendra virus ecology. Sci. Rep. 8 , 9555 (2018). 
36. Halpin, K. et al. Pteropid bats are confirmed as the reservoir hosts of henipaviruses: a comprehensive experimental study of virus transmission. Am. J. Trop. Med. Hyg. 85, 946-951 (2011).

37. Subudhi, S., Rapin, N. \& Misra, V. Immune system modulation and viral persistence in bats: understanding viral spillover. Viruses 11, 192 (2019).

38. Schuh, A. J. et al. Modelling filovirus maintenance in nature by experimental transmission of Marburg virus between Egyptian rousette bats. Nat. Commun 8, 14446 (2017).

Experimental demonstration of how Marburg virus transmits from infected to uninfected bats.

39. Glennon, E. E. et al. What is stirring in the reservoir? Modelling mechanisms of henipavirus circulation in fruit bat hosts. Philos. Trans. R. Soc. B Biol. Sci. 374 20190021 (2019).

40. O'Shea, T. J. et al. Bat flight and zoonotic viruses. Emerg. Infect. Dis. 20, 741-745 (2014).

41. Brook, C. E. \& Dobson, A. P. Bats as 'special' reservoirs for emerging zoonotic pathogens. Trends Microbiol. 23, 172-180 (2015)

42. Miller, M. R. et al. Broad and temperature independent replication potential of filoviruses on cells derived from old and new world bat species. J. Infect. Dis. 214 , S297-S302 (2016).

43. Paweska, J. T. et al. Virological and serological findings in Rousettus aegyptiacus experimentally inoculated with vero cells-adapted Hogan strain of Marburg virus. PLoS One 7, e45479 (2012).

44. Paweska, J. T. et al. Lack of Marburg virus transmission from experimentally infected to susceptible in-contact Egyptian fruit bats. J. Infect. Dis. 212, S109-S118 (2015)

45. Amman, B. R. et al. Oral shedding of Marburg virus in experimentally infected Egyptian fruit bats (Rousettus aegyptiacus). J. WildI. Dis. 51, 113-124 (2015).

46. Pawan, J. L. Rabies in the vampire bat of Trinidad, with special reference to the clinical course and the latency of infection. Ann. Trop. Med. Parasitol. 30, 401-422 (1936).

47. Schountz, T., Baker, M. L Butler J \& Munster, V. Immunological control of viral infections in bats and the emergence of viruses highly pathogenic to humans. Front. Immunol. 8, 1098 (2017)

48. Jones, M. E. B. et al. Clinical, histopathologic, and immunohistochemical characterization of experimental Marburg Virus Infection in a natural reservoir host, the Egyptian Rousette Bat (Rousettus aegyptiacus). Viruses 11, 214 (2019).

49. Kuzmin, I. V. et al. Innate immune responses of bat and human cells to Filoviruses: commonalities and distinctions. J. Virol. https://doi.org/10.1128/ JVI.02471-16 (2017)

50. Ahn, M. et al. Dampened NLRP3-mediated inflammation in bats and implications for a special viral reservoir host. Nat. Microbiol. 4, 789-799 (2019).

51. Wynne, J. W. et al. Comparative transcriptomics highlights the role of the activator protein transcription factor in the host response to Ebolavirus. J. Virol. https://doi.org/10.1128/JVI.01174-17 (2017).

52. Holzer, M. et al. Differential transcriptional responses to Ebola and Marburg virus infection in bat and human cells. Sci. Rep. 6, 34589 (2016).

53. Zhou, P. et al. Contraction of the type I IFN locus and unusual constitutive expression of IFN- $\alpha$ in bats. Proc. Natl Acad Sci. USA 113, 2696-2701 (2016).

54. Omatsu, T. et al. Induction and sequencing of Rousette bat interferon $\alpha$ and $\beta$ genes. Vet. Immunol. Immunopathol. 124, 169-176 (2008).

55. Pavlovich, S. S. et al. The Egyptian rousette genome reveals unexpected features of bat antiviral immunity. Cell 173, 1098-1110.e18 (2018)

56. Zhou, P. et al. Type III IFNs in pteropid bats: differential expression patterns provide evidence for distinct roles in antiviral immunity. J. Immunol. 186, 3138-3147 (2011)

57. Schuh, A. J. et al. Egyptian rousette bats maintain long-term protective immunity against Marburg virus infection despite diminished antibody levels. Sci. Rep. 7, 8763 (2017)

58. Jackson, F. R. et al. Experimental rabies virus infection of big brown bats (Eptesicus fuscus). J. WildI. Dis. 44, 612-621 (2008)

59. Schuh, A. J. et al. Antibody-mediated virus neutralization is not a universal mechanism of Marburg, Ebola, or Sosuga virus clearance in Egyptian rousette bats. J. Infect. Dis. 219, 1716-1721 (2019).

60. Middleton, D. J. et al. Experimental Nipah virus infection in pteropid bats (Pteropus poliocephalus). J. Comp. Pathol. 136, 266-272 (2007).
61. Turmelle, A. S., Jackson, F. R., Green, D. McCracken, G. F. \& Rupprecht, C. E. Host immunity to repeated rabies virus infection in big brown bats. J. Gen. Virol. 91, 2360-2366 (2010).

62. Katzourakis, A. \& Gifford, R. J. Endogenous viral elements in animal genomes. PLoS Genet. 6 , e1001191 (2010).

63. Menachery, V. D. et al. A SARS-like cluster of circulating bat coronaviruses shows potential for human emergence. Nat. Med. 21, 1508-1513 (2015).

64. Anthony, S. J. et al. Further evidence for bats as the evolutionary source of middle east respiratory syndrome coronavirus. mBio https://doi.org/10.1128/ mBio.00373-17 (2017).

65. Hoffmann, M. et al. Differential sensitivity of bat cells to infection by enveloped RNA viruses: coronaviruses, paramyxoviruses, filoviruses, and influenza viruses. PLoS One 8, e72942 (2013)

66. Lee, A. K. et al. De novo transcriptome reconstruction and annotation of the Egyptian rousette bat. BMC Genom. 16, 1033 (2015).

67. Teeling, E. C. et al. Bat biology, genomes, and the Bat $1 \mathrm{~K}$ project: to generate chromosome-leve genomes for all living bat species. Annu. Rev. Anim. Biosci. 6, 23-46 (2018).

68. Nikolay, B et al. Transmission of nipah virus - 14 years of investigations in Bangladesh. N. Engl. J. Med. 380, 1804-1814 (2019).

69. Selvey, L. A. et al. Infection of humans and horses by a newly described morbillivirus. Med. J. Aust. 162, 642-645 (1995).

70. Chua, K. B., Chua, B. H. \& Wang, C. W. Anthropogenic deforestation, El Nino and the emergence of Nipah virus in Malaysia. Malays. J. Pathol. 24, 15-21 (2002).

71. Guan, Y. et al. Isolation and characterization of viruses related to the SARS coronavirus from animals in southern China. Science 302, 276-278 (2003).

72. Zhou, P. et al. A pneumonia outbreak associated with a new coronavirus of probable bat origin. Nature $\mathbf{5 7 9}$ 270-273 (2020)

73. Pernet, O. et al. Evidence for henipavirus spillover into human populations in Africa. Nat. Commun. 5, 5342 (2014).

74. Chua, K. B. et al. A previously unknown reovirus of bat origin is associated with an acute respiratory disease in humans. Proc. Natl Acad. Sci. USA 104, 11424-11429 (2007).

75. Uehara, A. et al. Serological evidence of human infection by bat orthoreovirus in Singapore. J. Med. Virol. 91, 707-710 (2019).

76. Dovih, P. et al. Filovirus-reactive antibodies in humans and bats in Northeast India imply zoonotic spillover. PLoS Negl. Trop. Dis. 13, e0007733 (2019).

77. Wang, N. et al. Serological evidence of Bat SARS-related coronavirus infection in humans, China. Virol. Sin. 33 104-107 (2018)

\section{Evidence of additional spillover of lineage B} betacoronaviruses into humans after SARS-CoV but before SARS-CoV-2.

78. Howard, C. R. \& Fletcher, N. F. Emerging virus diseases: can we ever expect the unexpected? Emerg. Microbes Infect. 1, e46 (2012).

79. Brito, A. F. \& Pinney, J. W. Protein-protein interactions in virus-host systems. Front. Microbiol. 8, 1557 (2017).

80. Cockrell, A. S. et al. Mouse dipeptidyl peptidase 4 is not a functional receptor for Middle East respiratory syndrome coronavirus infection. J. Virol. 88, 5195-5199 (2014)

81. Li, W. et al. Receptor and viral determinants of SARS-coronavirus adaptation to human ACE2. EMBO J. 24, 1634-1643 (2005).

82. Mathewson, A. C. et al. Interaction of severe acute respiratory syndrome-coronavirus and NL63 coronavirus spike proteins with angiotensin converting enzyme-2. J. Gen. Virol. 89, 2741-2745 (2008).

83. Ndungo, E. et al. A single residue in Ebola virus receptor NPC1 influences cellular host range in reptiles. $m S$ phere https://doi.org/10.1128/ mSphere 00007-16 (2016).

84. Ng, M. et al. Filovirus receptor NPC1 contributes to species-specific patterns of ebolavirus susceptibility in bats. elife 4, e11785 (2015)

85. Letko, M. et al. Adaptive evolution of MERS-CoV to species variation in DPP4. Cell Rep. 24, 1730-1737 (2018).

86. Batra, J. et al. Protein interaction mapping identifies RBBP6 as a negative regulator of Ebola virus replication. Cell 175, 1917-1930.e13 (2018).
87. Shah, P. S. et al. Comparative flavivirus-host protein interaction mapping reveals mechanisms of Dengue and Zika virus pathogenesis. Cell 175, 1931-1945. e18 (2018)

88. Martinez-Gil, L., Vera-Velasco, N. M. \& Mingarro, I. Exploring the human-Nipah virus protein-protein interactome. J. Virol. 91, e01461-17 (2017).

89. Tripathi, S. et al. Meta- and orthogonal integration of influenza "OMICs" data defines a role for UBR4 in virus budding. Cell Host Microbe 18, 723-735 (2015).

90. Streicker, D. G. et al. Host phylogeny constrains cross-species emergence and establishment of rabies virus in bats. Science 329, 676-679 (2010).

91. Plowright, R. K. et al. Pathways to zoonotic spillover Nat. Rev. Microbiol. 15, 502-510 (2017).

92. Bonaparte, M. I. et al. Ephrin-B2 ligand is a functiona receptor for Hendra virus and Nipah virus. Proc. Natl Acad. Sci. USA 102, 10652-10657 (2005)

93. Negrete, O. A. et al. EphrinB2 is the entry receptor for Nipah virus, an emergent deadly paramyxovirus. Nature 436, 401-405 (2005)

94. Negrete, O. A. et al. Two key residues in ephrinB3 are critical for its use as an alternative receptor for Nipah virus. PLoS Pathog. 2, e7 (2006).

95. Carette, J. E. et al. Ebola virus entry requires the cholesterol transporter Niemann-Pick C1. Nature 477, 340-343 (2011).

96. Cote, M. et al. Small molecule inhibitors reveal Niemann-Pick C1 is essential for Ebola virus infection. Nature 477, 344-348 (2011).

97. $\mathrm{Li}, \mathrm{W}$. et al. Angiotensin-converting enzyme 2 is a functional receptor for the SARS coronavirus. Nature 426, 450-454 (2003)

98. Raj, V. S. et al. Dipeptidyl peptidase 4 is a functional receptor for the emerging human coronavirus-EMC. Nature 495, 251-254 (2013).

99. Wan, Y., Shang, J., Graham, R., Baric, R. S. \& Li, F. Receptor recognition by novel coronavirus from Wuhan: an analysis based on decade-long structural studies of SARS coronavirus. J. Virol. 94, e00127-20 (2020).

100. Peck, K. M. et al. Glycosylation of mouse DPP4 plays a role in inhibiting middle East respiratory syndrome coronavirus infection. J. Virol. 89 , 4696-4699 (2015).

101. Li, F. Receptor recognition and cross-species infection of SARS coronavirus. Antivir. Res. 100, 246-254 (2013).

102. Allison, A. B. et al. Host-specific parvovirus evolution in nature is recapitulated by in vitro adaptation to different carnivore species. PLoS Pathog. 10 , e1004475 (2014)

103. Moncla, L. H. et al. Selective bottlenecks shape evolutionary pathways taken during mammalian adaptation of a 1918-like avian influenza virus. Cell Host Microbe 19, 169-180 (2016).

104. Smith, E. C. \& Denison, M. R. Coronaviruses as DNA wannabes: a new model for the regulation of RNA virus replication fidelity. PLoS Pathog. 9, e1003760 (2013).

105. Ogando, N. S et al. The curious case of the nidovirus exoribonuclease: its role in RNA synthesis and replication fidelity. Front. Microbiol. 10, 1813 (2019).

106. Graepel, K. W. et al. Proofreading-deficient coronaviruses adapt for increased fitness over long-term passage without reversion of exoribonuclease-inactivating mutations. $\mathrm{mBio}$ https://doi.org/10.1101/175562 (2017).

107. Ferron, F. et al. Structural and molecular basis of mismatch correction and ribavirin excision from coronavirus RNA. Proc. Natl Acad. Sci. USA 115 E162-E171 (2018)

108. Denison, M. R., Graham, R. L., Donaldson, E. F., Eckerle, L. D. \& Baric, R. S. Coronaviruses: an RNA proofreading machine regulates replication fidelity and diversity. RNA Biol. 8, 270-279 (2011).

109. Zhang, Z., Shen, L. \& Gu, X. Evolutionary dynamics of MERS-CoV: potential recombination, positive selection and transmission. Sci. Rep. 6, 25049 (2016).

110. Wang, Y. et al. Origin and possible genetic recombination of the middle east respiratory syndrome coronavirus from the first imported case in china: phylogenetics and coalescence analysis. $\mathrm{mBio}$ 6, e01280-15 (2015)

111. Sabir, J. S. et al. Co-circulation of three came coronavirus species and recombination of MERS-CoVs in Saudi Arabia. Science 351, 81-84 (2016).

112. Dudas, G. \& Rambaut, A. MERS-CoV recombination: implications about the reservoir and potential for adaptation. Virus Evol. 2, vev023 (2016). 
113. Lau, S. K. et al. Severe acute respiratory syndrome (SARS) coronavirus ORF8 protein is acquired from SARS-Related coronavirus from greater horseshoe bats through recombination. J. Virol. 89, 10532-10547 (2015).

114. Hu, B. et al. Discovery of a rich gene pool of bat SARS-related coronaviruses provides new insights into the origin of SARS coronavirus. PLoS Pathog. 13, e1006698 (2017).

115. Ding, N. Z., Xu, D. S., Sun, Y. Y., He, H. B. \& He, C. Q. A permanent host shift of rabies virus from Chiroptera to Carnivora associated with recombination. Sci. Rep. 7, 289 (2017)

116. Simon, V., Bloch, N. \& Landau, N. R. Intrinsic host restrictions to HIV-1 and mechanisms of viral escape Nat. Immunol. 16, 546-553 (2015).

117. Krupp, A. et al. APOBEC3G polymorphism as a selective barrier to cross-species transmission and emergence of pathogenic SIV and AIDS in a primate host. PLoS Pathog. 9, e1003641 (2013)

118. Etienne, L. et al. The role of the antiviral APOBEC3 gene family in protecting chimpanzees against lentiviruses from monkeys. PLoS Pathog. 11, e 1005149 (2015).

119. James, J. et al. Influenza A virus PB1-F2 protein prolongs viral shedding in chickens lengthening the transmission window. J. Gen. Virol. 97, 2516-2527 (2016).

120. van Doremalen, N. et al. SARS-like coronavirus WIV1-CoV does not replicate in Egyptian fruit bats (Rousettus aegyptiacus). Viruses 10, 727 (2018).

121. Paweska, J. T. et al. Experimental inoculation of Egyptian fruit bats (Rousettus aegyptiacus) with Ebola virus. Viruses 8, 29 (2016).

122. Bharaj, P. et al. The host E3-ubiquitin ligase TRIM6 ubiquitinates the Ebola virus VP35 protein and promotes virus replication. J. Virol. https://doi.org/ 10.1128/JVI.00833-17 (2017).

123. Sakuma, T., Noda, T., Urata, S., Kawaoka, Y. \& Yasuda, J. Inhibition of Lassa and Marburg virus production by tetherin. J. Virol. 83, 2382-2385 (2009).

124. Kaletsky, R. L., Francica, J. R., Agrawal-Gamse, C. \& Bates, P. Tetherin-mediated restriction of filovirus budding is antagonized by the Ebola glycoprotein. Proc. Natl Acad. Sci. USA 106, 2886-2891 (2009).

125. Jouvenet, N. et al. Broad-spectrum inhibition of retroviral and filoviral particle release by tetherin J. Virol. 83, 1837-1844 (2009).

126. Huang, I. C. et al. Distinct patterns of IFITM-mediated restriction of filoviruses, SARS coronavirus, and influenza a virus. PLoS Pathog. 7, e 1001258 (2011)

127. Hoffmann, M. et al. Tetherin inhibits Nipah virus but not Ebola virus replication in fruit bat cells. J. Virol. 93, e01821-18 (2019).

128. Marceau, C. D. et al. Genetic dissection of Flaviviridae host factors through genome-scale CRISPR screens. Nature 535, 159-163 (2016)

129. Zhang, R. et al. A CRISPR screen defines a signal peptide processing pathway required by flaviviruses. Nature 535, 164-168 (2016).

130. Dukhovny, A et al. A CRISPR activation screen identifies genes protecting from Zika virus infection J. Virol. 93, e00211-19 (2019).

131. Park, R. J. et al. A genome-wide CRISPR screen identifies a restricted set of HIV host dependency factors. Nat. Genet. 49, 193-203 (2017).

132. Ma, Y. et al. CRISPR/Cas9 screens reveal epstein-barr virus-transformed $\mathrm{B}$ cell host dependency factors. Cell Host Microbe 21, 580-591.e7 (2017).

133. Han, J. et al. Genome-wide CRISPR/Cas9 Screen Identifies host factors essential for influenza virus replication. Cell Rep. 23, 596-607 (2018).

134. Heaton, B. E. et al. A CRISPR activation screen identifies a pan-avian influenza virus inhibitory host factor. Cell Rep. 20, 1503-1512 (2017).

135. Anthony, S. J. et al. Global patterns in coronavirus diversity. Virus Evol. 3, vex012 (2017).

136. Becker, M. M. et al. Synthetic recombinant bat SARS-like coronavirus is infectious in cultured cells and in mice. Proc. Natl Acad. Sci. USA 105, 19944-19949 (2008).

137. Marsh, G. A. et al. Cedar virus: a novel Henipavirus isolated from Australian bats. PLoS Pathog. 8 , e 1002836 (2012).

138. Laing, E. D. et al. Rescue and characterization of recombinant cedar virus, a non-pathogenic Henipavirus species. Virol. J. 15, 56 (2018).
139. Zhang, C. et al. Seroreactive profiling of filoviruses in Chinese bats reveals extensive infection of diverse viruses. J. Virol. https://doi.org/10.1128/ JVI.02042-19 (2020)

140. Letko, M., Marzi, A. \& Munster, V. Functional assessment of cell entry and receptor usage for SARS-CoV-2 and other lineage B betacoronaviruses. Nat. Microbiol. 5, 562-569 (2020). An example of an approach to functionally testing many related viruses in parallel, as outlined in Box 3.

141. Kan, B. et al. Molecular evolution analysis and geographic investigation of severe acute respiratory syndrome coronavirus-like virus in palm civets at an animal market and on farms. J. Virol. 79, 11892-11900 (2005).

142. Adney, D. R. et al. Replication and shedding of MERS-CoV in upper respiratory tract of inoculated dromedary camels. Emerg. Infect. Dis. 20, 1999-2005 (2014).

143. Euren, J. et al. Living Safely with Bats. https://www. ecohealthalliance.org/wp-content/uploads/2018/10/ Living-Safely-with-Bats_download.pdf (EcoHealth Alliance, 2018)

144. Kessler, M. K. et al. Changing resource landscapes and spillover of henipaviruses. Ann. N. Y. Acad. Sci. 1429, 78-99 (2018).

145. Warimwe, G. M. et al. Chimpanzee Adenovirus vaccine provides multispecies protection against rift valley fever. Sci. Rep. 6, 20617 (2016)

146. van Doremalen, N. et al. A single-dose ChAdOx 1vectored vaccine provides complete protection against Nipah Bangladesh and Malaysia in Syrian golden hamsters. PLoS Negl. Trop. Dis. 13, e0007462 (2019).

147. Munster, V. J. et al. Protective efficacy of a novel simian adenovirus vaccine against lethal MERS-CoV challenge in a transgenic human DPP4 mouse model. NPJ Vaccines 2, 28 (2017).

148. Broder, C. C., Weir, D. L. \& Reid, P. A. Hendra virus and Nipah virus animal vaccines. Vaccine 34 , 3525-3534 (2016)

149. Dowd, K. A. et al. Rapid development of a DNA vaccine for Zika virus. Science 354, 237-240 (2016).

150. Luby, S. P. et al. Foodborne transmission of Nipah virus, Bangladesh. Emerg. Infect. Dis. 12, 1888-1894 (2006).

151. Khan, S. U. et al. A randomized controlled trial of interventions to impede date palm sap contamination by bats to prevent nipah virus transmission in Bangladesh. PLoS One 7, e42689 (2012).

152. Kuisma, E. et al. Long-term wildlife mortality surveillance in northern Congo: a model for the detection of Ebola virus disease epizootics. Philos. Trans. R. Soc. Lond. B Biol. Sci. 374, 20180339 (2019).

153. Mahl, P. et al. Twenty year experience of the oral rabies vaccine SAG2 in wildlife: a global review. Vet. Res. 45, 77 (2014)

154. Almeida, M. F., Martorelli, L. F., Aires, C. C. Barros, R. F. \& Massad, E. Vaccinating the vampire bat Desmodus rotundus against rabies. Virus Res. 137, 275-277 (2008)

155. Mencher, J. S. et al. Protection of black-tailed prairie dogs (Cynomys ludovicianus) against plague after voluntary consumption of baits containing recombinant raccoon poxvirus vaccine. Infect. Immun. 72, 5502-5505 (2004)

156. Middleton, D. et al. Hendra virus vaccine, a one health approach to protecting horse, human, and environmental health. Emerg. Infect. Dis. 20 , 372-379 (2014)

157. Field, H. E. Hendra virus ecology and transmission. Curr. Opin. Virol. 16, 120-125 (2016).

158. Munster, V. J. et al. Outbreaks in a rapidly changing central Africa - lessons from Ebola. N. Engl. J. Med. 379, 1198-1201 (2018)

159. Wang, L. et al. Emergence and control of infectious diseases in China. Lancet 372, 1598-1605 (2008).

160. Plowright, R. K., Becker, D. J., McCallum, H. \& Manlove, K. R. Sampling to elucidate the dynamics of infections in reservoir hosts. Philos. Trans. $R$. Soc. Lond. B Biol. Sci. 374, 20180336 (2019)

161. Peel, A. J. et al. The effect of seasonal birth pulses on pathogen persistence in wild mammal populations. Proc. Biol. Sci. 281, 20132962 (2014).
162. Plowright, R. K. et al. Reproduction and nutritional stress are risk factors for Hendra virus infection in little red flying foxes (Pteropus scapulatus). Proc. Biol. Sci. 275, 861-869 (2008).

163. Rahman, M. A. et al. Date palm sap linked to Nipah virus outbreak in Bangladesh, 2008. Vector Borne Zoonotic Dis. 12, 65-72 (2012).

164. Luby, S. P. et al. Recurrent zoonotic transmission of Nipah virus into humans, Bangladesh, 2001-2007 Emerg. Infect. Dis. 15, 1229-1235 (2009).

165. Field, H. et al. Spatiotemporal aspects of hendra virus infection in pteropid bats (Flying-Foxes) in Eastern Australia. PLoS One 10, e0144055 (2015).

166. Paez, D. J. et al. Conditions affecting the timing and magnitude of Hendra virus shedding across pteropodid bat populations in Australia. Epidemiol. Infect. 145 3143-3153 (2017)

167. Plowright, R. K. et al. Prioritizing surveillance of Nipah virus in India. PLoS Negl. Trop. Dis. 13, e0007393 (2019).

168. Han, B. A. et al. Undiscovered bat hosts of filoviruses. PLoS Negl. Trop. Dis. 10, e0004815 (2016).

169. Albery, G., Eskew, A. E., Ross, N. \& Olival, K. J. Predicting the global mammalian viral sharing network using phylogeography. Nat. Commun. 11, 2260 (2020).

170. Schountz, T. Immunology of bats and their viruses: challenges and opportunities. Viruses 6, 4880-4901 (2014).

171. Reid, J. E. \& Jackson, A. C. Experimental rabies virus infection in Artibeus jamaicensis bats with CVS-24 variants. J. Neurovirol 7, 511-517 (2001).

172. Munster, V. J. et al. Replication and shedding of MERS-CoV in Jamaican fruit bats (Artibeus jamaicensis). Sci. Rep. 6, 21878 (2016).

173. Malmlov, A. et al. Experimental Zika virus infection of Jamaican fruit bats (Artibeus jamaicensis) and possible entry of virus into brain via activated microglial cells. PLoS Negl. Trop. Dis. 13, e0007071 (2019).

174. Jones, M. E. et al. Experimental inoculation of Egyptian Rousette bats (Rousettus aegyptiacus) with viruses of the Ebolavirus and Marburgvirus genera. Viruses 7, 3420-3442 (2015).

175. Suu-Ire, R. et al. Pathogenesis of bat rabies in a natural reservoir: comparative susceptibility of the straw-colored fruit bat (Eidolon helvum) to three strains of Lagos bat virus. PLoS Negl. Trop. Dis. 12, e0006311 (2018)

176. Cogswell-Hawkinson, A. et al. Tacaribe virus causes fatal infection of an ostensible reservoir host, the Jamaican fruit bat. J. Virol. 86, 5791-5799 (2012).

177. Phelps, K. L. et al. Bat research networks and viral surveillance: gaps and opportunities in Western Asia. Viruses 11, 240 (2019).

\section{Acknowledgements}

This work was supported (in part) by the Intramural Research Program of the NIH. V.J.M. and R.P. are supported by the DARPA PREEMPT Program Cooperative Agreement (No. D18AC00031). K.J.O. is supported by the USAID Emerging Pandemic Threats PREDICT program, NIH/NIAID award R01Al110964 and a US Defense Threat Reduction Agency award HDTRA11710064.

\section{Author contributions}

The authors contributed equally to all aspects of the article.

Competing interests

The authors declare no competing interests.

Publisher's note

Springer Nature remains neutral with regard to jurisdictional claims in published maps and institutional affiliations.

\section{RELATED LINKS}

Database of bat-associated viruses: http://www.mgc.ac.cn/

Novel 2019 coronavirus on virological.org:

https://virological.org/c/novel-2019-coronavirus/

(c) This is a U.S. government work and not under copyright protection in the U.S.; foreign copyright protection may apply. 2020 